www.ccsenet.org/journal.html

\title{
A Note on Two Theorems of C. Dong and J. Wang \\ Concerning Combinatorial Identities
}

\author{
Arnold R. Kräuter \\ Department of Mathematics and Information Technology \\ University of Leoben \\ Franz-Josef-Strasse 18, A-8700 Leoben, Austria
}

Tel: 43-3842-402-3803 E-mail: kraeuter@unileoben.ac.at

\section{Abstract}

In a recent paper C. Dong and J. Wang rederived three classical combinatorial identities by applying a special Vandermonde determinant. Two of their results, however, turn out to be incorrectly stated. This note presents counterexamples along with revised versions of the results mentioned.
\end{abstract}

Keywords: Vandermonde determinant, Identities involving binomial coefficients

\section{Introduction}

C. Dong and J. Wang applied a special Vandermonde determinant in order to establish a couple of well-known combinatorial identities in an elementary way (Dong \& Wang, 2007). Unfortunately, two of these are incorrectly stated. In the following we present counterexamples as well as revised versions of the respective theorems in the cited paper.

Let $V_{n}$ denote the $n$-square Vandermonde matrix (cf. Lancaster \& Tismenetsky, 1985, p. 35, Exercise 6) of the integers $1,2, \ldots, n$,

$$
V_{n}=\left[\begin{array}{ccccc}
1 & 1 & 1 & \cdots & 1 \\
1 & 2 & 3 & \cdots & n \\
1 & 2^{2} & 3^{2} & \cdots & n^{2} \\
\vdots & \vdots & \vdots & \ddots & \vdots \\
1 & 2^{n-1} & 3^{n-1} & \cdots & n^{n-1}
\end{array}\right],
$$

and let $D_{n}=\operatorname{det}\left(V_{n}\right)$ be the determinant of $V_{n}$. Furthermore, let $M_{j}$ be the minor of the entry in the $n$th row and $j$ th column of $V_{n}$, and let $S_{j}$ be the minor of the entry in the first row and $j$ th column of $V_{n}$.

Lemma 1 (Dong \& Wang, 2007, p. 24, Eq. (1)).

$$
M_{j}=\left(\prod_{m=1}^{n-2} m !\right)\left(\begin{array}{l}
n-1 \\
n-j
\end{array}\right), \quad j=1,2, \ldots, n .
$$

Lemma 2 (Dong \& Wang, 2007, p. 24, Eq. (2)).

$$
S_{j}=\left(\prod_{m=1}^{n-1} m !\right)\left(\begin{array}{l}
n \\
j
\end{array}\right), \quad j=1,2, \ldots, n .
$$

Using (1) and (2) Dong and Wang obtained, among others, the following identities for positive integers $n$ (Dong \& Wang, 2007, p. 25, Eqs. (3) and (6), resp.):

$$
\sum_{j=1}^{n}(-1)^{n+j} j^{n-1}\left(\begin{array}{l}
n \\
j
\end{array}\right)=n !
$$




$$
\sum_{j=0}^{n-1}(-1)^{j}(n-j)^{i}\left(\begin{array}{c}
n \\
j
\end{array}\right)=0, \quad 1 \leq i \leq n,
$$

\section{Counterexamples to Eqs. (3) and (4)}

In their current form, Eqs. (3) and (4) turn out to be incorrect.

Example 1. Eq. (3) is false. For if, e. g., $n=4$, (3) would imply

$$
\sum_{j=1}^{4}(-1)^{4+j} j^{3}\left(\begin{array}{l}
4 \\
j
\end{array}\right)=-4+48-108+64=0 \neq 4 ! .
$$

Example 2. Eq. (4) is false in the case $i=n$. For if, e. g., $i=n=4$, (4) would imply

$$
\sum_{j=0}^{3}(-1)^{j}(4-j)^{4}\left(\begin{array}{l}
4 \\
j
\end{array}\right)=256-324+96-4=24 \neq 0
$$

\section{Restatements of Eqs. (3) and (4) with proofs}

The original Eqs. (3) and (4) have to be replaced by the following statements.

Theorem 1 (cf. Gould, 1972, p. 2, Eq. (1.13), first part). For every nonnegative integer $n$ we have

$$
\sum_{j=1}^{n}(-1)^{n+j} j^{n}\left(\begin{array}{l}
n \\
j
\end{array}\right)=n !
$$

Proof. According to the original proof (Dong \& Wang, 2007, p. 25, Theorem 1) the following holds:

$$
D_{n}=\sum_{j=1}^{n}(-1)^{n+j} j^{n-1}\left(\prod_{m=1}^{n-2} m !\right)\left(\begin{array}{c}
n-1 \\
n-j
\end{array}\right)=\prod_{m=1}^{n-1} m !
$$

This gives

$$
\sum_{j=1}^{n}(-1)^{n+j} j^{n-1}\left(\begin{array}{c}
n-1 \\
n-j
\end{array}\right)=(n-1) !
$$

Using the identity (cf. Gould, 1972, p. iv)

$$
\left(\begin{array}{c}
n-1 \\
n-j
\end{array}\right)=\frac{j}{n}\left(\begin{array}{c}
n \\
j
\end{array}\right), \quad 1 \leq j \leq n
$$

we eventually obtain (5).

Theorem 2 (cf. Gould, 1972, p. 2, Eq. (1.13), second part). For every nonnegative integer $n$ we have

$$
\sum_{j=0}^{n-1}(-1)^{j}(n-j)^{i}\left(\begin{array}{c}
n \\
j
\end{array}\right)=0, \quad 1 \leq i \leq n-1 .
$$

Proof. Let $W_{n}^{(i)}$ denote the $n$-square matrix obtained from the Vandermonde matrix $V_{n}$ by replacing the $n$th row by the $i$ th row, $1 \leq i \leq n-1$, 


$$
W_{n}^{(i)}=\left[\begin{array}{ccccc}
1 & 1 & 1 & \cdots & 1 \\
1 & 2 & 3 & \cdots & n \\
\vdots & \vdots & \vdots & \ddots & \vdots \\
1 & 2^{n-2} & 3^{n-2} & \cdots & n^{n-2} \\
1 & 2^{i-1} & 3^{i-1} & \cdots & n^{i-1}
\end{array}\right] .
$$

Since $W_{n}^{(i)}$ is singular by construction, expansion of $\operatorname{det}\left(W_{n}^{(i)}\right) \quad$ by the $n$th row gives

$$
0=\operatorname{det}\left(W_{n}^{(i)}\right)=\sum_{j=1}^{n}(-1)^{n+j} j^{i-1} M_{j}
$$

Using Lemma 1 and Eq. (6) we get

$$
\begin{aligned}
& 0=\sum_{j=1}^{n}(-1)^{n+j} j^{i-1}\left(\prod_{m=1}^{n-2} m !\right)\left(\begin{array}{c}
n-1 \\
n-j
\end{array}\right)= \\
& =\sum_{j=1}^{n}(-1)^{n+j} j^{i}\left(\prod_{m=1}^{n-2} m !\right) \frac{1}{n}\left(\begin{array}{l}
n \\
j
\end{array}\right) .
\end{aligned}
$$

Replacing $j$ by $n-j$ and by the symmetry of the binomial coefficients we eventually obtain (7).

Remark. The arguments used in the proof of Theorem 2 still hold when $W_{n}^{(i)}$ is replaced by the matrix

$$
X_{n}^{(i)}=\left[\begin{array}{ccccc}
1 & 2^{i-1} & 3^{i-1} & \cdots & n^{i-1} \\
1 & 2 & 3 & \cdots & n \\
\vdots & \vdots & \vdots & \ddots & \vdots \\
1 & 2^{n-2} & 3^{n-2} & \cdots & n^{n-2} \\
1 & 2^{n-1} & 3^{n-1} & \cdots & n^{n-1}
\end{array}\right], \quad 2 \leq i \leq n,
$$

followed by the expansion of $\operatorname{det}\left(X_{n}^{(i)}\right)$ by the first row (which involves the numbers $S_{j}$ given in Lemma 2).

\section{References}

Dong, Changzhou, \& Wang, Junqing (2007). Application of Vandermonde determinant in combination mathematics. Modern Applied Science, 1 (2), 24 - 26.

Gould, Henry W. (1972). Combinatorial Identities. Revised Edition. Morgantown, WV: H. W. Gould.

Lancaster, Peter, \& Tismenetsky, Miron (1985). The Theory of Matrices. Second Edition with Applications. Orlando, FL: Academic Press. 